\title{
Skin Necrosis with Oculomotor Nerve Palsy Due to a Hyaluronic Acid Filler Injection
}

\author{
Jae Il Lee, Seok Joo Kang, Hook Sun \\ Department of Plastic and Reconstructive Surgery, Busan Baik Hospital, InJe University School of Medicine, Busan, Korea
}

Performing rhinoplasty using filler injections, which improve facial wrinkles or soft tissues, is relatively inexpensive. However, intravascular filler injections can cause severe complications, such as skin necrosis and visual loss. We describe a case of blepharoptosis and skin necrosis caused by augmentation rhinoplasty and we discuss the patient's clinical progress. We describe the case of a 25-year-old female patient who experienced severe pain, blepharoptosis, and decreased visual acuity immediately after receiving a filler injection. Our case suggests that surgeons should be aware of nasal vascularity before performing an operation, and that they should avoid injecting fillers at a high pressure and/or in excessive amounts. Additionally, filler injections should be stopped if the patient complains of severe pain, and appropriate measures should be taken to prevent complications caused by intravascular filler injections.

Keywords Dermal fillers / Hyaluronic Acid / Necrosis
Correspondence: Seok Joo Kang Department of Plastic and Reconstructive Surgery, Busan Baik Hospital, InJe University School of Medicine, 75 Bokji-ro, Busanjin-gu, Busan 47392, Korea

Tel: +82-51-890-6136

Fax: +82-51-894-7976

E-mail: sonydr@naver.com

Received: 25 Oct $2016 \bullet$ Revised: 25 Jan 2017 • Accepted: 27 Feb 2017

pISSN: 2234-6163 • elSSN: 2234-6171 • https://doi.org/10.5999/aps.2017.44.4.340 • Arch Plast Surg 2017;44:340-343

No potential conflict of interest relevant to this article was reported.

\section{INTRODUCTION}

Performing rhinoplasty using filler injections, which improve facial wrinkles or soft tissues, is relatively inexpensive. However, intravascular filler injections can cause severe complications, such as skin necrosis and visual loss $[1,2]$. We describe a case of blepharoptosis and skin necrosis caused by augmentation rhinoplasty and we discuss the patient's clinical progress. In this case, ocular ischemic palsy occurred immediately after an intravascular filler was injected; thus, we discuss how to determine whether an intravascular filler injection is a cause of ocular ischemic palsy. This brief clinical study also presents an appropriate treatment strategy with the goal of minimizing complications based on the progress of ocular ischemic palsy.

\section{CASE}

This study was reviewed and approved by the Ethics Review Board of the InJe University Health Center.

A 25-year-old female patient was admitted as an outpatient to the Plastic Surgery Department of our hospital due to experiencing severe pain, blepharoptosis, and decreased visual acuity immediately after receiving a filler injection. At the local clinic, the patient was injected with hyaluronidase promptly after the filler injection. The orbital pain and dizziness appeared 3 hours before admission; that is, during augmentation rhinoplasty with hyaluronic acid (Bellast, Dongkook Pharm. Co., Seoul, Korea).

On admission, ecchymosis on the left nasal ridge, ptosis of the left eye, a conjunctival injection, and a subtly dilated pupil in the left eye were observed. She experienced diplopia in all direc- 
tions, and extraocular movement was limited to a right gaze.

Color change and edema were found in the territories of the superior trochlear artery and angular artery, such as the forehead, nasal tip, and medial side of the orbit (Fig. 1).

There were no significant findings on magnetic resonance imaging or brain angiography. Partial retinal ischemia was observed on fundoscopy (Fig. 2).

Systemic steroid injections were administered for 2 weeks, and broad-spectrum antibiotics were administered for 1 week. The skin lesion was dressed twice per day with an epidermal growth factor spray and antibacterial ointment. One week later, she had

\section{Fig. 1. Photograph: on the day of admission}

$(A, B)$ Color change and edema were observed in the territories of the Superior trochlear artery and angular artery, such as the forehead, nasal tip, and medial side of the orbit.
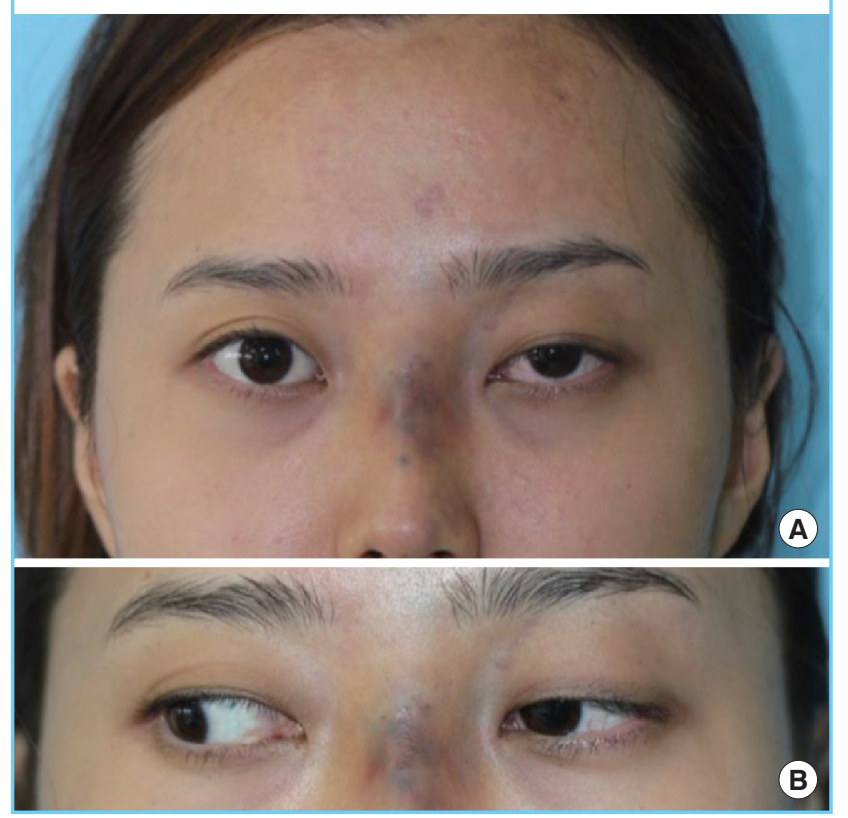

\section{Fig. 2. Fundoscopy image}

Partial retinal ischemia was shown on fundoscopy (black arrows).

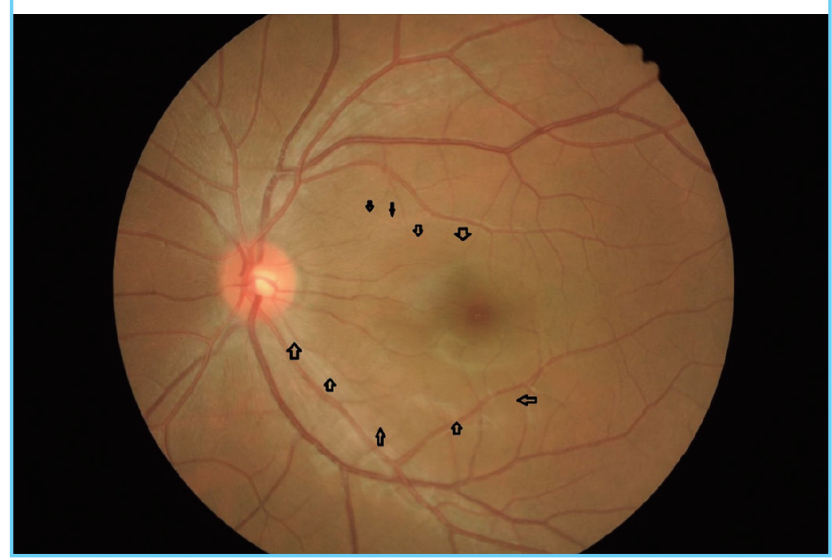

almost recovered from blepharoptosis and her limited extraocular movement had improved. The skin lesion was aggravated for 5 days but it progressively improved (Fig. 3 ).

Only redness remained after 2 weeks (Fig. 4). By 6 months, the skin lesion had improved with barely any scarring, and her persistent diplopia progressively resolved (Fig. 5).

\section{Fig. 3. Photograph after 1 week of treatment \\ (A, B) Systemic steroid injections were administered for 2 weeks, and broad-spectrum antibiotics were administered for 1 week. The skin lesion became aggravated for 5 days, but it progressively improved.}
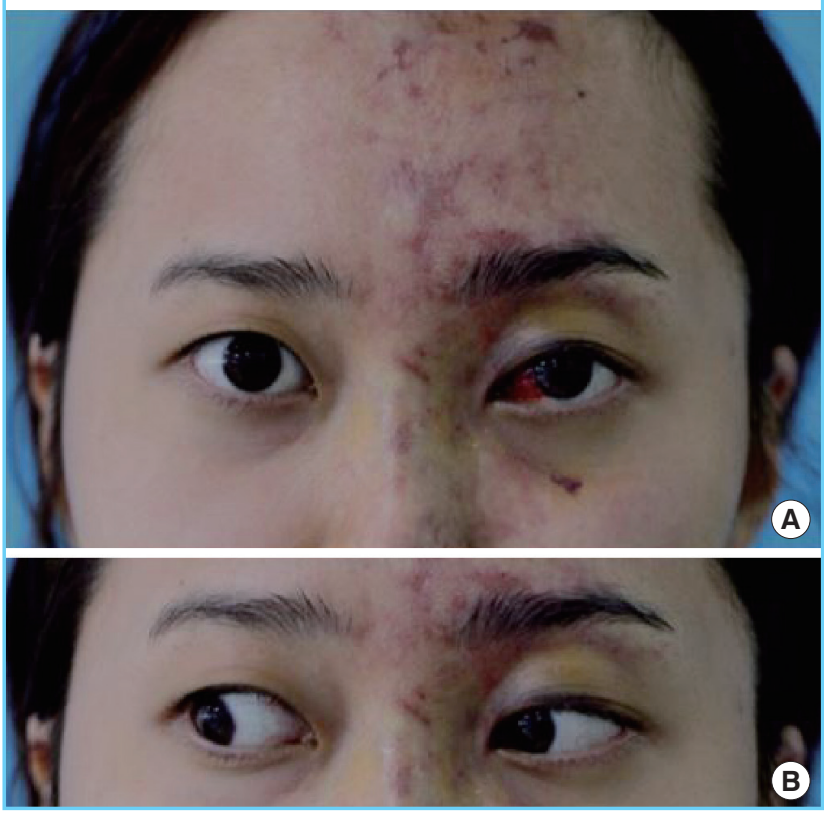

Fig. 4. Photograph after 2 weeks of treatment

$(A, B)$ Only redness remained on the skin lesion.
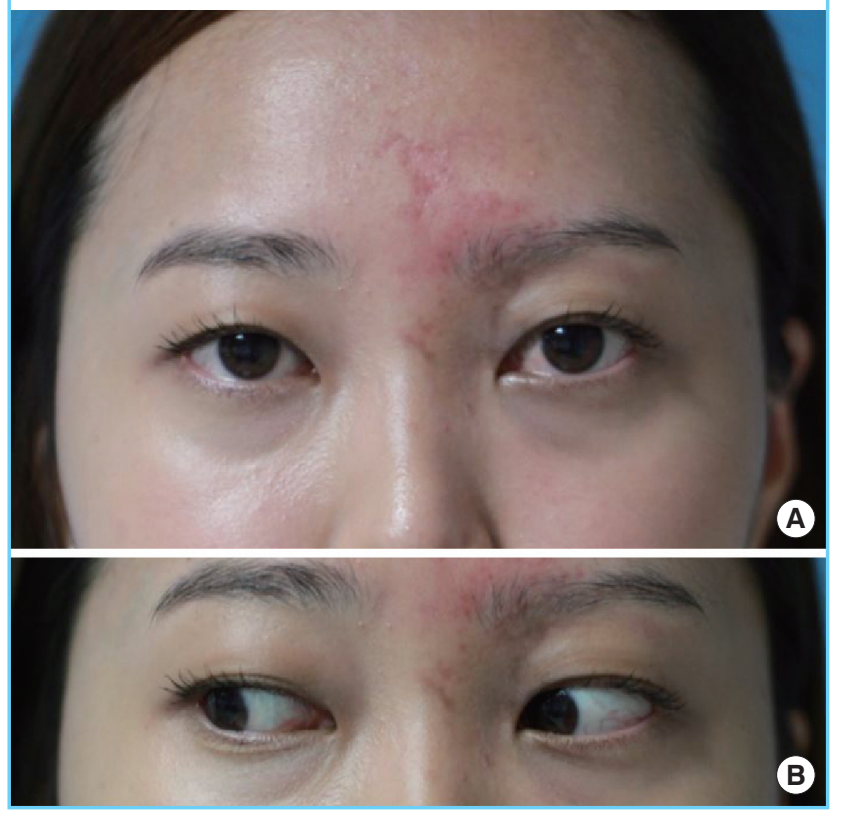


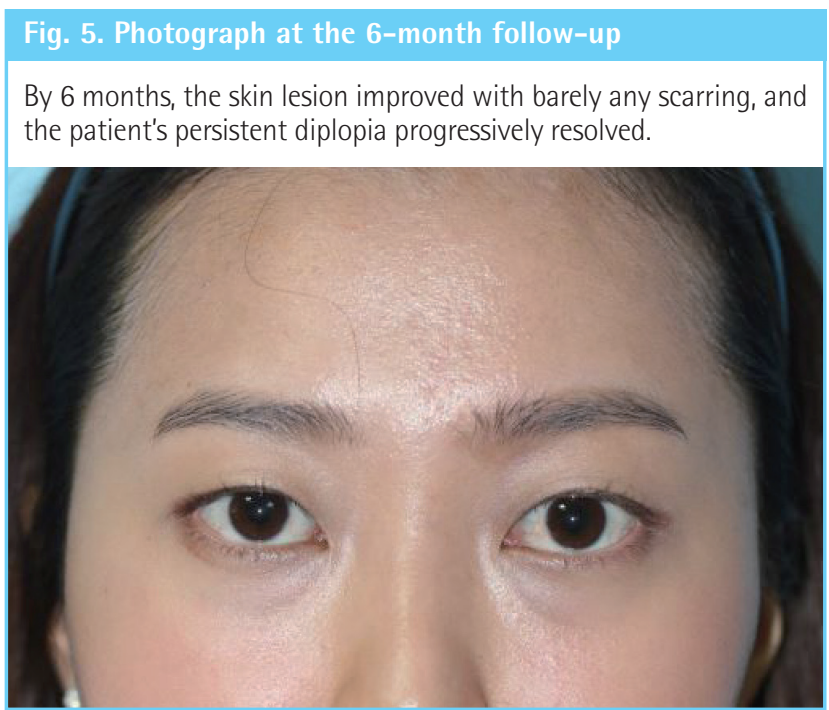

\section{DISCUSSION}

In the present case, the fact that the surgeon immediately recognized that the filler was injected into the blood vessel by accident and observed the subsequent clinical symptoms was important. If the patient's clinical progress could have been predicted, appropriate treatment could have minimized the complications. Although skin necrosis and oculomotor nerve palsy are uncommon, they are the most serious complications of augmentation rhinoplasty with filler injections [2-4].

Ischemic injuries are caused when small particles of the filler are injected into the blood vessels. Since adipose tissue particles have a range of sizes, they can clot blood vessels of various sizes. However, hyaluronic acid products such as Restylane (Q-Med, Uppsala, Sweden) are unlikely to clot the ophthalmic artery, which is $2 \mathrm{~mm}$ in diameter, since they contain gel particles that are $400 \mu \mathrm{m}$ in diameter [5].

Additionally, it is noteworthy that the occlusion in our case occurred during augmentation rhinoplasty rather than during wrinkle treatment of the glabella, which is associated with a high risk of encountering a blood vessel. There are many distal branches of the ophthalmic artery on the nasal ridge and glabella. We infer that the ischemic injury in the present case was caused by filler particles that reached the ophthalmic artery through the dorsal nasal artery during augmentation rhinoplasty. The unexpected complications were caused by a combination of factors, including the distinctive vascularity around the nose, excessive filler injection pressure, and excessive filler injection volume.

Our patient presented with nausea, vomiting, a headache, and swelling of the treatment area immediately after receiving an intravascular filler injection. Three hours later, blepharoptosis was observed. The ecchymosis on her face became gradually aggravated, causing blisters on day 4 , and discoloration reached its peak on day 7. Although the skin lesion remained aggravated until day 7 , the patient recovered from blepharoptosis. Her facial skin lesion gradually improved, and she achieved an almost full recovery after 2 months. As shown in the present case, appropriate treatment with steroids and antibiotics will first improve ptosis, and improvement in the skin will occur subsequently.

Several precautions should be taken to avoid complications caused by an intravascular filler injection. Regurgitation should always be performed before a filler is injected, and blunt needles or blunt cannulas should be used instead of a sharp needle $[4,5]$. Injecting filler at a high pressure and/or in a large volume should be avoided, and the filler injection should be stopped as soon as the patient complains of a toothache or headache. Immediate warm gauze compression to the proper area can help restore the blood supply, through rapid subsequent vasodilatation [6].

Additionally, nitroglycerin paste is available to facilitate more significant vasodilation, and low- molecular-weight heparin helps prevent thrombosis and embolism [7].

Management of erythema and reddish swelling with steroids and Vitamin Kis also effective [8].

With the increased demand for filler injections, the occurrence of unexpected complications is also increasing. Surgeons should be aware of nasal vascularity before performing an operation, and they should avoid injecting fillers at a high pressure and/or in excessive volumes [6]. Additionally, filler injections should be stopped if the patient complains of severe pain, and appropriate measures should be taken to prevent complications caused by intravascular filler injections. In conclusion, it is necessary to immediately perform the appropriate treatment for ocular ischemic palsy occurring due to a filler injection based on the clinical progress of the injury in order to minimize complications.

\section{REFERENCES}

1. Peter S, Mennel S. Retinal branch artery occlusion following injection of hyaluronic acid (Restylane). Clin Exp Ophthalmol 2006;34:363-4.

2. Bachmann F, Erdmann R, Hartmann V, et al. The spectrum of adverse reactions after treatment with injectable fillers in the glabellar region: results from the Injectable Filler Safety Study. Dermatol Surg 2009;35 Suppl 2:1629-34.

3. Danesh-Meyer HV, Savino PJ, Sergott RC. Case reports and small case series: ocular and cerebral ischemia following facial injection of autologous fat. Arch Ophthalmol 2001;119: 777-8.

4. Cohen JL. Understanding, avoiding, and managing dermal 
filler complications. Dermatol Surg 2008;34 Suppl 1:S92-9.

5. Glaich AS, Cohen JL, Goldberg LH. Injection necrosis of the glabella: protocol for prevention and treatment after use of dermal fillers. Dermatol Surg 2006;32:276-81.

6. Hanke CW, Higley HR, Jolivette DM, et al. Abscess formation and local necrosis after treatment with Zyderm or Zyplast collagen implant. J Am Acad Dermatol 1991;25:319-26.
7. Schanz S, Schippert W, Ulmer A, et al. Arterial embolization caused by injection of hyaluronic acid (Restylane). Br J Dermatol 2002;146:928-9.

8. Cohen JL, Bhatia AC. The role of topical vitamin K oxide gel in the resolution of postprocedural purpura. J Drugs Dermatol 2009;8:1020-4. 